\title{
EGRET HIGH-ENERGY GAMMA-RAY OBSERVATIONS OF AGN: ENERGY SPECTRA AND TIME VARIABILITY
}

\author{
D.J. THOMPSON, D.L. BERTSCH, B.L. DINGUS*, \\ J. A. ESPOSITO*, C.E. FICHTEL, R.C. HARTMAN, \\ S.D. HUNTER, P. SREEKUMAR* \\ NASA/Goddard Space Flight Center \\ Greenbelt, Maryland 20771 USA
}

J. CHIANG, J. M. FIERRO, Y.C. LIN, P.F. MICHELSON, P.L. NOLAN Stanford University Stanford, California, 94305 USA

\section{G. KANBACH, H.A. MAYER-HASSELWANDER, C.V.MONTIGNY} H.-D. RADECKE

Max Planck Institut für Extraterrestrische Physik

Giessenbachstr, 85748 Garching Germany

\section{D.A. KNIFFEN}

Hampden-Sydney College

Hampden-Sydney, Virginia, 23943 USA

\section{J.R. MATTOX operated by Astronomy Programs, Computer Sciences Corporation Greenbelt, MD 20770 USA \\ E.J. SCHNEID \\ Grumman Aerospace Corporation \\ Bethpage, New York 11714 USA}

Compton Observatory Science Support Center,

\begin{abstract}
The Energetic Gamma Ray Experiment Telescope (EGRET) on the Compton Gamma Ray Observatory has detected more than 20 Active Galactic Nuclei (AGN) at photon energies above $30 \mathrm{MeV}$.
\end{abstract}

\section{Introduction}

Since the launch of the Compton Gamma Ray Observatory in April, 1991, EGRET has been mapping the high energy gamma ray sky. During the all-sky survey and in the

*USRA Research Associate

49

T. J.-L. Courvoisier and A. Blecha: Multi-Wavelength Continuum Emission of AGN, 49-52.

(C) 1994 IAU. Printed in the Netherlands. 
observations since then, EGRET has identified 26 gamma-ray-bright AGN. All were detected with statistical significance of $5 \sigma$ or greater.

\section{Identifications}

Although EGRET source error boxes are relatively large (ranging from a few arcminutes to more than a degree in diameter), the number of high-energy gamma ray sources at high latitudes is modest; therefore, source confusion is not a serious problem. Gamma rays in the $100 \mathrm{MeV}$ energy range are also inherently nonthermal, requiring high energy particles as their progenitors. The search for counterparts can focus on likely sites of particle acceleration. In the case of the EGRET sources, a very high fraction (more than $70 \%$ ) of the high galactic latitude error boxes contain one particular type of AGN. These are characterized by their radio properties, particularly in the 1-5 GHz band: radio bright (more than $0.5 \mathrm{Jy}$ ), flat or nearly flat spectrum $(\alpha>-0.6)$, and core dominated. An extrapolation of the Kühr et al. (1981) catalog indicates that there are fewer than 900 such sources in the sky. Such objects are generally thought to have jets, which provide the likely sites of particle acceleration to produce the gamma rays. Table 1 lists the EGRET detections, along with properties which indicate that many of these have characteristics of blazars.

\section{Energy Spectra}

Over at least the central part of the EGRET energy range (50 MeV to several GeV), all the AGN energy spectra can be well represented by power laws. As shown in Table 1 , the photon number spectra range from -1.5 to -2.6 . Combining the EGRET results with those from other frequencies (usually not contemporaneous) produces multifrequency spectra such as the example of Fig. 1. The gamma rays represent a major contributor to the total observed power of the source. In this example, the simultaneous EGRET (Hunter et al., 1993) and COMPTEL (Collmar, et al., 1993) observations show strong evidence for a spectral break near $10^{22} \mathrm{~Hz}$.

\section{Time Variability}

Most of the EGRET-detected AGN show time variability on scales of days to months. An example is shown in Fig. 2. In addition to supporting the concept of a jet origin for the gamma radiation, the variability provides an important tool for modeling the physical processes in these jets. Correlations and time lags between gamma-ray flares and those seen at other wavelengths are crucial tests of models.

\section{References}

Bertsch, D.L. et al.: 1993, 'Detection of Gamma-Ray Emission from the Quasar PKS 0208-512', ApJ 405, pp. L21-L24.

Collmar, W. et al.: 1993, 'Preliminary COMPTEL Results on the Quasar PKS 0528+134', Proc. 23rd Inter. Cosmic Ray Conf. (Calgary), 1, pp. 168-171.

Hunter, S.D. et al.: 1993, 'Detection of High-Energy Gamma Rays from Quasar PKS 0528+134 by EGRET on the Compton Gamma Ray Observatory', ApJ 409, pp. 134-138.

Kühr, H., Witzel, A., Pauliny-Toth, I.I.K., and Nauber, U.: 1991, 'A Catalogue of Extragalactic Radio Sources Having Flux Densities Greater than $1 \mathrm{Jy}$ at 5 GHz', $A \& A S u p p l$ 45, pp. 367-430. 


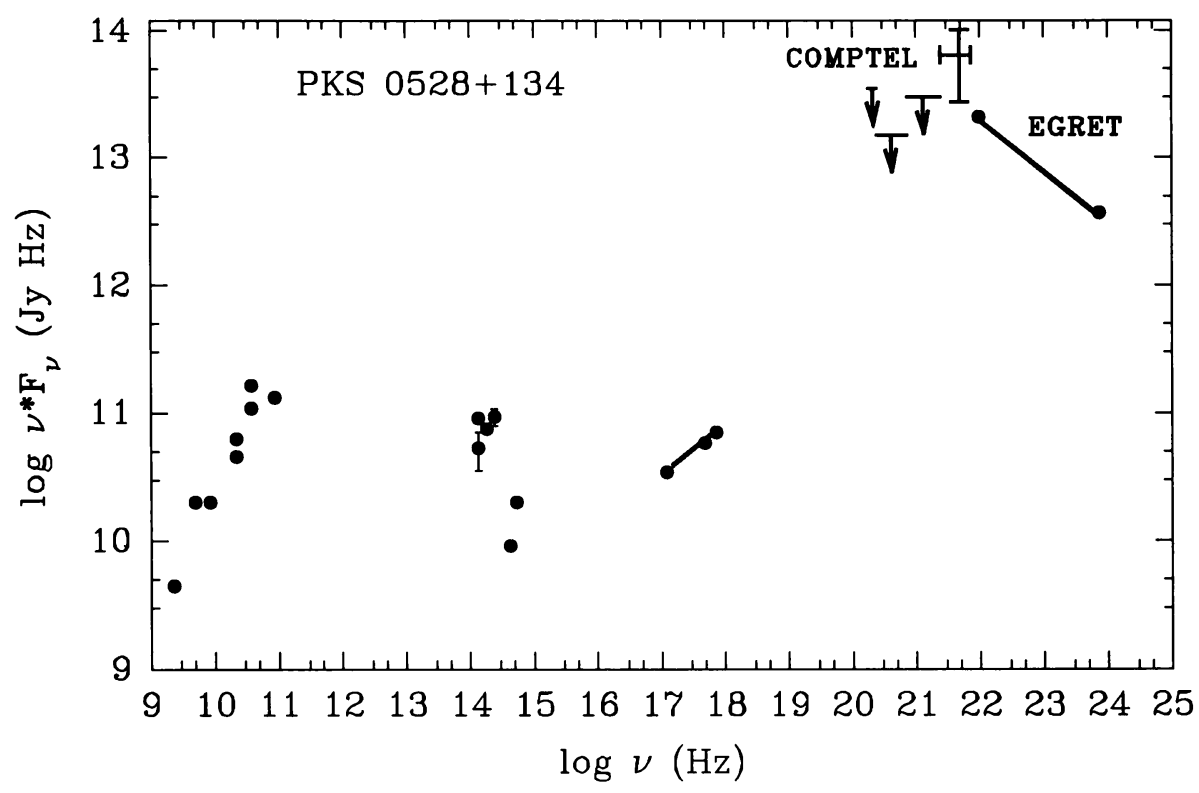

Fig. 1 - Multifrequency spectrum of PKS 0528+134. The EGRET (Hunter et al., 1993) and COMPTEL (Collmar et al., 1993) observations were made in May, 1991. References to other observations are given by Hunter et al.

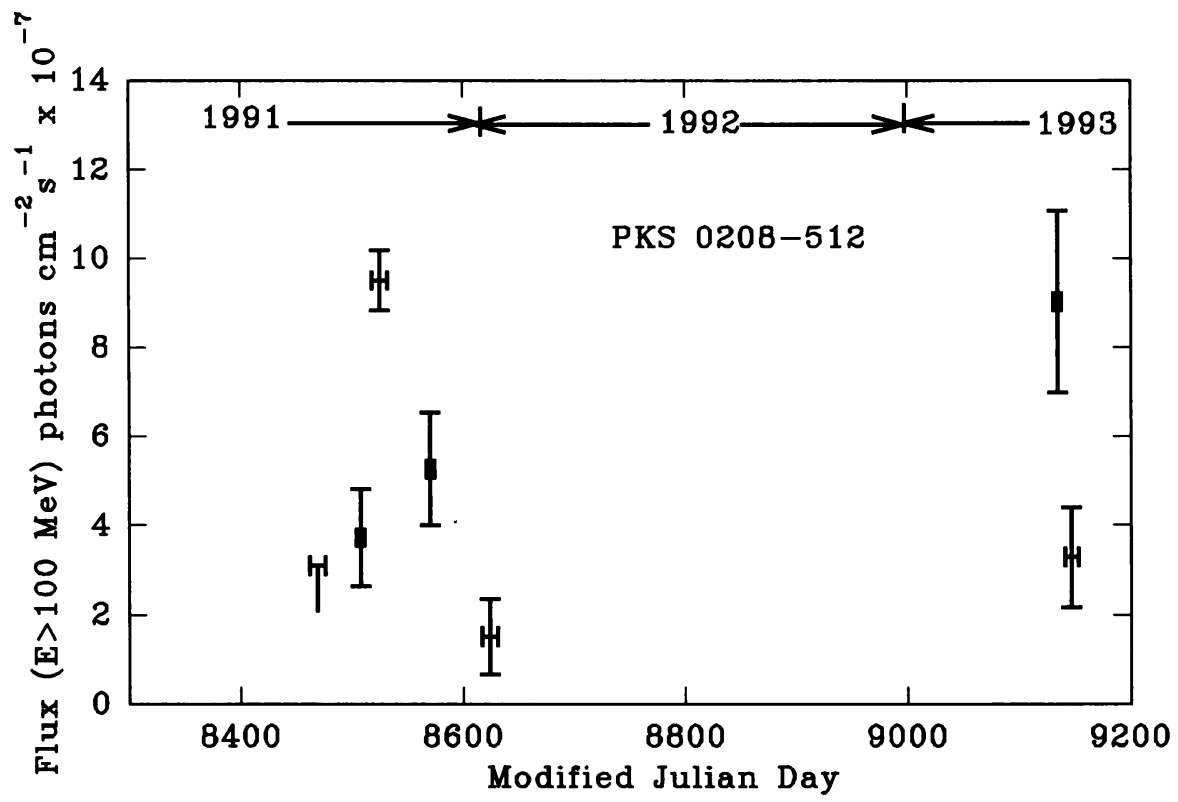

Fig. 2 -- Time variability of $\mathrm{E}>100 \mathrm{MeV}$ gamma rays from PKS 0208-512 as seen by EGRET. Data points from 1991-1992 are from Bertsch et al. (1993). 


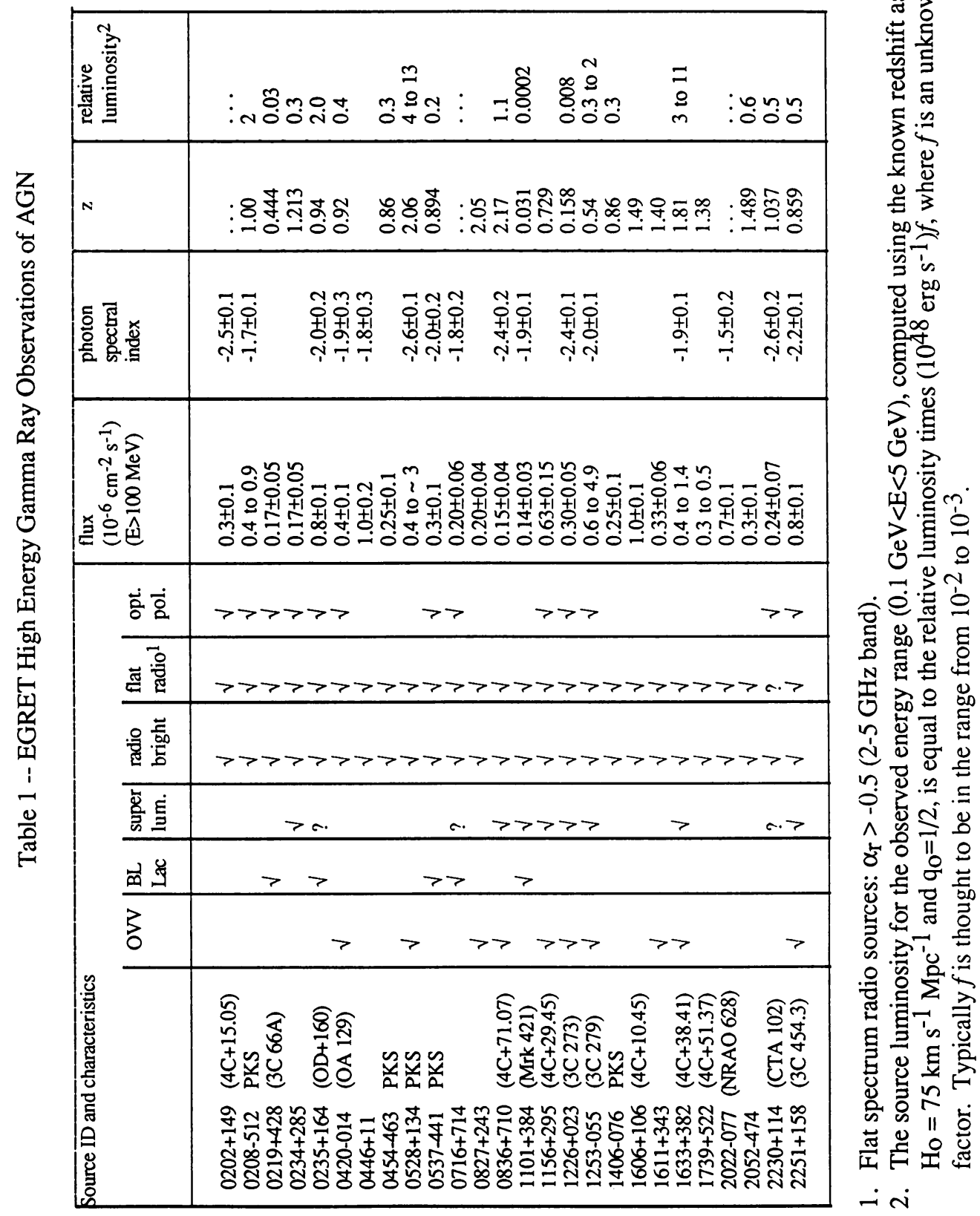

